\title{
REFLEXIONES EN TORNO A LA METODOLOGÍA DE LA HISTORIA DE LA FILOSOFÍA
}

LAURa Benítez

Instituto de Investigaciones Filosóficas, UNAM

\section{Introducción}

La pregunta sobre el status filosófico de la historia de la filosofía no ha sido nunca una pregunta de fácil solución. En efecto, en el siglo pasado, bajo el imperio del historicismo, la historia de la filosofía no resultaba sino una parte de la historia general humana y como tal su tratamiento metodológico no se distinguía del de la historia de los otros aspectos de la cultura humana.

En ese mismo siglo, Hegel puso de manifiesto algunos de los problemas peculiares de esta disciplina al reflexionar sobre la tensión que existe entre los términos historia (como lo cambiante) y filosofía (como lo permanente) y dio solución a esa contradicción asentando el carácter dinámico de la filosofía en la continuidad evolutiva de los sistemas. La historia de la filosofía vino entonces a representar un canon, un parámetro para la selección de filósofos o propuestas filosóficas. A este respecto la elección hegeliana fue muy clara, sólo los sistemas pueden ser considerados como filosofía y cualquier historia de la filosofía que no manifieste con claridad el criterio de selección de lo filosófico no es sino un agregado erudito de datos.

El reclamo hegeliano puso de manifiesto que la historia de la filosofía no era una historia sin más. El compromiso metodológico, al margen del idealismo, implica, en este caso, la completa reconstrucción racional del acaecer filosófico. Naturalmente, este proceder, que ya Aristóteles había empleado, en alguna medida, al hablar de la filosofía griega anterior a él, hace ver que la historia de la filosofía no está limitada a la reconstrucción histórica, sea ésta de carácter externo y contextual o de carácter interno genético, siguiendo la producción y desarrollo de los conceptos.

Toda reconstrucción racional involucra criterios evaluativos sobre la actividad filosófica que se historia y van desde pretender que se cuenta con el criterio que permite distinguir entre lo verdaderamente filosófico y lo que no lo es, hasta otros menos pretenciosos que ayudan a una selección de lo filosófico, ya por la vigencia de una 
problemática, ya por la cercanía con nuestros intereses, necesidades, etc,. En todo caso, desde el punto de vista metodológico, parece haber una anterioridad natural de tales criterios de selección de lo "filosófico" (con mayúsculas o minúsculas) que pueden ser leídos como guía racional con respecto a la reconstrucción histórica.

Preocupada por diversos aspectos metodológicos de la filosofía y por los compromisos que conllevan los métodos más comunes para la elaboración de la historia de la filosofía, considero oportuno señalar que está fuera de cuestión la reflexión filosófica sobre la metodología de la historia de la filosofía. Esta tarea metodológica y epistemológica a que se sujeta prácticamente todo saber, es reconocida y prestigiosamente filosófica. Lo que está hoy en tela de juicio es si la tarea de historiar la filosofía es o no una tarea filosófica.

1. ¿Es la historia de la filosofia un saber histórico o un saber filosófico?

El preguntar por la historia de la filosofía como auténtico objeto filosófico obliga a delimitar, de manera inmediata, el campo de estudio de la filosofía; sin embargo, es justo en la historia de la filosofía donde, a partir de alguna perspectiva teórica, se ponen en ejercicio los criterios que permiten distinguir lo filosófico de lo que no lo es.

Buena parte de la labor de los estudiosos, los intérpretes, los comentaristas y manualistas consiste en valorar a los autores y decirnos qué y cómo leerlos. Naturalmente que media una enorme distancia entre quien ofrece la discusión sobre un argumento de Descartes, quien ofrece una nueva lectura de todo el sistema platónico o quien ambiciona reconstruir racionalmente todo el saber filosófico; no obstante, es claro que, de manera general, quien historia la filosofía tiene en mente una idea de lo que ésta debe ser y decide sobre la pertinencia de tópicos y autores con arreglo a la norma o guía racional de su elección.

Es verdad que en muchas ocasiones nos encontramos con historias de la filosofía que no exhiben ni criterios de selección, ni perspectivas o justificaciones para la presentación de los más disímbolos temas o autores. Se trata de ficheros de datos reductivos e incoloros cuyo autor brilla por su ausencia; caricaturas incapaces de proporcionar idea alguna acerca del saber filosófico.

Queda entonces claro, espero, que de manera inicial para hacer historia de la filosofía se requiere de una perspectiva filosófica que permita la selección y ordenación racional de aquello que se va a reconstruir históricamente.

Por otra parte, si la labor es historiar, se espera que la historia de la filosofía, como la de cualquier otro campo de la cultura, se sujete 
a las pautas metodológicas de cualquier elaboración histórica. El conocimiento de las diversas modalidades historiográficas permite al historiador de la filosofía el dar el enfoque más adecuado al objeto de estudio que ha constituido bajo el modelo de filosofía que considera mejor. Hago énfasis en el criterio de selección de lo filosófico porque ello ayuda a entender por qué la historia de la filosofía no es una historia sin más $y$, en consecuencia, por qué no se trata de un puro objeto histórico. Lo filosófico pesa aquí, de entrada, en el marco teórico como guía racional y no sólo como contenido.

Otra razón por la que molesta la consideración de que la historia de la filosofía es un puro objeto histórico es que si bien es a todas luces indispensable tomar en cuenta las determinaciones externas que explican el desarrollo histórico de la filosofía, no es menos importante para la historiografía filosófica la consideración del desarrollo de las ideas y de cierta lógica interna que ilumina su comprensión. Sin embargo, esa lógica interna está también íntimamente ligada al punto de vista sobre lo filosófico que se haya adoptado y por ello rige la interpretación de los sucesos filosóficos buscando dar sentido y unidad a su presentación.

Tal parece, entonces, que la lógica interna del desarrollo filosófico no puede explicarse exclusivamente por las determinaciones externas, esto es, con la reconstrucción histórico-contextual del saber filosófico sino que depende en buena medida de la guía racional o parámetro normativo sobre lo filosófico que hará posible, en muy diversos grados, según los objetivos de los historiadores, la reconstrucción racional de la filosofía.

\section{Algunos señalamientos sobre reconstrucción histórica}

Desde la perspectiva histórica la presentación de los sucesos filosóficos va, en el siglo pasado, de la presentación escueta de los hechos, esto es, "dejar que hablen los autores", sustentada en las doctrinas de la plena objetividad y el mayor acopio de material, entre paréntesis, posición erudita de cuño positivista, a la interpretación, como un tipo de reconstrucción racional que, en el caso del idealismo hegeliano, se lleva a cabo bajo las premisas de la unidad y universalidad del pensamiento filosófico. Tal pretensión cedió su lugar a la explicación en la historia de la filosofia como una forma de interpretación más compleja y enriquecida que, a partir del descubrimiento de ciertas regularidades del desarrollo histórico se considera capaz de dar cuenta al propio tiempo del desarrollo de las diversas formas de la cultura, incluida, naturalmente, la filosófica.

Con sus diversas modalidades estos modelos de reconstrucción histórica hacen hincapié en el hecho de que el objetivo principal de la 
historia de la filosofía es decir lo que el autor dijo, contextualizando, naturalmente, ese decir en su propia época, aunque no se limiten a ello.

Esta meta, que cada vez me aparece más como un ideal regulativo de la reconstrucción histórica en la filosofía, plantea sin duda muchas dificultades, pero quiero restringirme únicamente a la siguiente cuestión: ¿Hasta qué punto es posible para quien historia la filosofía el decir lo que el autor dijo sin traducirlo (léase interpretarlo) a sus propios términos, bajo sus propios marcos teóricos y con su propio instrumental metodológico? Es posible, o resulta de algún interés no valorar o enfatizar ciertos aspectos del pensamiento de un autor de acuerdo con nuestra propia problemática o necesidades actuales.

En mi opinión es por estas razones de enfoque y traducción por las que la historia de la filosofía no puede ser nunca una pura tarea histórica. El hilo de la explicación tiene que ir más allá del orden cronológico de la presentación de los sucesos y más allá, también, de la explicación en función de las condiciones generales externas. La interpretación en la historia de la filosofía tiene que tener, como Jano, una cara vuelta hacia las condiciones externas y concretas que la determinan y otra hacia su dinamismo y estructura interna; pero no basta con ello, en efecto, casi todo historiador de la filosofía resiente la necesidad de dialogar con los autores que presenta; para ello enjuicia, valora, recorta y retoma lo que considera de mayor interés, más certero o viable, más positivo o aleccionador, etc. para su momento. ¿Es esto hacer violencia a un autor? ¿Es realmente decir lo que no dijo? ¿Es realmente una labor pedante y anacrónica que se opone por completo a la reconstrucción histórica?

\section{Algunas reflexiones sobre las reconstrucciones racionales}

Cuando en este siglo la filosofía analítica puso entre paréntesis la actividad de historiar la filosofía como verdaderamente filosófica llevando esta propuesta hasta sus últimas consecuencias, se produjeron ciertos cismas que han dado lugar a interesantes polémicas.

John Yolton, campeón de la reconstrucción histórica contextual, mostró, en un foro sobre estas cuestiones hace cuatro años, no sólo la importancia formativa de la historia de la filosofía, sino la importancia filosófica de la historia de la filosofía.

La parte contraria enfatizaba que lo importante es hacer filosofía y que los filósofos anteriores no son sino buenos pretextos para plantear de manera polémica las propias propuestas.

En esa misma línea, Bernard Williams en el prólogo a su Descartes. El proyecto de la investigación pura se suma a esta idea y la 
pone en práctica. Para él, un texto de historia de la filosofía interesante debe ser más un texto de filosofía, esto es, un texto en el cual se discuta con el autor del pasado en los términos de la problemática presente, haciendo uso de la metodología actual y restringiéndose a los temas vigentes. En suma, el diálogo interesante del vivo con el muerto parece darle más peso al vivo y ser más irrespetuoso con el muerto.

Aquí, la pregunta que surge naturalmente es si puede considerarse esta clase de diálogo como auténtica historia de la filosofía. Este problema tiene mucho que ver con los límites entre la reconstrucción histórica y la racional. De otra manera, ies posible a la reconstrucción histórica prescindir de una perspectiva, una metodología, juicios de valor, etc., frente a los autores que estudia? Y si estos aspectos no son prescindibles, ¿hasta qué punto son compatibles con la misma?

Por otra parte, ¿es posible a la reconstrucción racional dialogar al margen y con absoluta independencia del sistema sobre los contenidos teóricos y argumentos de un autor? ¿Es plenamente prescindible el contexto histórico cuando se pretenden valorar afirmaciones y argumentos?

Al parecer estas dos formas de reconstrucción no son excluyentes sino hasta cierto punto complementarias. No podemos hacer historia de la filosofía sin tomar en cuenta a los autores y su tiempo, con ignorancia absoluta de las condiciones concretas, externas e internas, que dan lugar a su peculiar filosofar; pero a la par, no parece posible historiar una época, escuela o autor, sin que medie un diálogo de nuestra parte con los muertos, diálogo en el que afloran nuestro conocimiento del instrumental metodológico, los intereses y preocupaciones que nos son contemporáneos y, en fin, nuestros juicios, preferencias y valoraciones.

Richard Rorty llega a la conclusión de que las reconstrucciones racionales e históricas son necesarias para la historia de la filosofía pero no forman parte de una misma labor intelectual. Para él, hay que hacer ambas cosas pero separadamente. Así, habrá quien se especialice en la reconstrucción racional y quien lo haga en la reconstrucción histórica. 1

Mi opinión, por el contrario, es que se requieren mutuamente y que en mayor o menor grado ambas perspectivas se tocan siempre al hacer historia de la filosofía. Queda pues por elucidar esta mayor o menor medida tratando de establecer en la confluencia de estos

1 Richard Rorty, J. B. Schneewind y Quentin Skinner, Philosophy in History: Essays on the Historiography of Philosophy, Cambridge University Press, Cambridge, 1984. 
métodos cuáles serían las proporciones más gratificantes y exitosas y por qué razones.

\section{Relaciones entre reconstrucción racional e histórica}

Como ya referimos, algunos autores analíticos suelen ver una oposición insalvable entre la reconstrucción histórica y la racional. Richard Rorty, por ejemplo, sostiene que quien se dedica a la reconstrucción histórica usa una metodología completamente diferente de quien se dedica a la reconstrucción racional, ya que la del primero es una tarea meramente erudita de recopilación que da prioridad a la ubicación contextual, relativizando el valor de verdad de las teorías en sí mismas, pues se insiste en que dicho valor debe verse en función del sistema de creencias de la época (científicas y religiosas principalmente). Por su parte, quien reconstruye racionalmente una teoría considera que realiza una labor auténticamente filosófica ya que únicamente usa al autor del pasado como un interlocutor a quien se interroga sobre los problemas y preocupaciones presentes, tratando de establecer el valor absoluto, ahistórico de sus teorías. La fuerza demostrativa de los argumentos debe valer por sí misma al margen de las creencias, los problemas concretos y el sistema filosófico en que se originan.

El reconstructor racional, desde esta corriente de pensamiento, se ve a sí mismo en posesión de un método preciso e incontaminado, un instrumental aséptico que le permite decidir sobre el valor de verdad de las muestras lógicas analizadas, limpiamente recortadas de un tiempo y de un espacio y trasplantadas a otro completamente ajeno en el que, normalmente, no suelen encajar.

Desde esta perspectiva los dos métodos de reconstrucción aludidos no resultan conciliables; sin embargo, existen otras alternativas.

La pregunta que surge es: ¿acaso la reconstrucción racional se limita a la descripción de los procedimientos de la filosofía analítica?

En la actualidad se han desarrollado otras posiciones que apuntan hacia la unión de reconstrucción racional e histórica. Charles Taylor en su artículo "Philosophy of History" y Alasdair Macintyre en "The Relationship of Philosophy to its Past", proponen el término reconstrucción creativa que refiere de alguna manera a esta síntesis. ${ }^{2}$

Sin llegar al extremo de Hegel de decir que la filosofía es su historia, Taylor considera que una de las tareas fundamentales de la filosofía es encontrar las raíces de nuestras concepciones, ideologías y creencias, lo cual sólo puede hacerse a través de la historia de

2 Charles Taylor en Richard Rorty et. al., op. cit.; Alasdair Macintyre en Richard Rorty et al., op. cit. 
la filosofía. La idea fundamental es que como pueblos con cultura, como grupos humanos o sujetos individuales, los seres humanos necesitamos de esa memoria histórica que nos permite tener una mejor comprensión de nuestro presente y, en alguna forma, oriente nuestras acciones futuras. Idea que, por lo demás, se ha manifestado en distintos momentos del desarrollo del pensamiento humano.

Parece que hoy día la filosofía anglosajona está más atenta o reconsidera las posibilidades de explicación que ofrece la historia de la filosofía, pues aun el propio Rorty, aunque no ve la posibilidad de unir la reconstrucción histórica a la racional, considera que ambas tareas son importantes.

En esta línea y de manera más definida, en un artículo de 1965 ("The Idea of History of Philosophy"), dirigido contra la afirmación kantiana de que la filosofía no es su historia, John Passmore hace algunas precisiones muy importantes para los efectos de esta presentación.

Es verdad que el modo de hacer historia de la filosofía puede resultar en un producto no filosófico si se enfatiza de tal manera la continuidad de los problemas que se concluya que en filosofía no hay nada nuevo bajo el sol, esto es, que toda la tarea del historiador se reduzca a reordenar lo que ya ha sido dicho una y otra vez.

Por su parte, los filósofos subrayan demasiado su originalidad y creatividad como si en efecto hubiera una absoluta discontinuidad entre unos y otros.

Ciertamente la enfermedad profesional del filósofo es exagerar su propia originalidad; probablemente la enfermedad profesional del historiador sea insistir demasiado fuertemente en la continuidad. ${ }^{3}$

La propuesta de Passmore es una salida intermedia. El buen historiador debe estar atento tanto a las continuidades como a las discontinuidades. La idea de fondo es que sólo puede hablarse de historia de la filosofía si se supone una relativa continuidad de clases de problemas en que se dan rupturas. No todo puede ser radicalmente nuevo, pero sí hay novedades. En efecto, no hay un objeto fijo de la filosofía, no hay un conjunto definido e intocable de problemas puesto que hay la posibilidad de que surjan otros nuevos, lo que hay son clases de problemas recurrentes en la filosofía. Asimismo, por lo que hace a las soluciones, los filósofos no siempre dan soluciones sino que hacen más claros los problemas al mostrar sus implicaciones,

3 John Passmore, "The Idea of History of Philosophy" en History and Theory, Mouton and Co. Printers, The Hague, 1965, p. 3. 
incluso nos dicen por qué son insostenibles algunas soluciones aparentemente plausibles, etc. En suma, lo que los filósofos ofrecen, en general, es un avance en la comprensión de los problemas. Por otra parte, Passmore asimila bajo el rubro de historia polémica lo que Rorty ha llamado reconstrucción racional y la caracteriza siguiendo a Broad como:

... estudiar las soluciones que hombres de genio han dado a un problema filosófico cuyos puntos de vista difieran unos de otros lo más posible. El choque de sus opiniones encenderá una luz que nos capacite para evaluar los errores en los que cayeron, y al darnos cuenta de los aspectos fuertes y débiles de cada teoría descubriremos la dirección en la cual puede darse un progreso futuro. ${ }^{4}$

Para algunos autores, esta forma de acceder a los filósofos del pasado tiene el defecto de que no es historia sino filosofía ya que se hace crítica filosófica al tratar de elucidar si las teorías son o no razonables.

Aquí resurge el problema de la continuidad y la discontinuidad. En efecto, quien hace historia polémica prescinde de la temporalidad. Todas las teorías se sitúan en un mismo tiempo; en esa perspectiva los problemas filosóficos no cambian. El historiador insistiría más en que cada época tiene sus peculiares problemas filosóficos, éstos varían de tiempo en tiempo y de lugar en lugar. Passmore no hace suya esta tesis de Collingwood ni niega, como éste lo hace, la posibilidad de una historia polémica de la filosofía.

Nuevamente, la idea de Passmore es que hay esta especie de continuidad relativa. Es verdad que los polemistas exageran la modernidad de los filósofos, pero no es menos cierto que los historiadores exageran su pertenencia a una cultura específica como si nada pudiera permearse de una a otra. La solución es un saludable término medio. Así, la fórmula productiva o valiosa que permite poner en contacto a la reconstrucción histórica con la racional, que es el problema medular de la historia de la filosofía, consiste en que los estudios polémicos son buenos sólo si se fundan en una comprensión histórica razonable.

No parece haber ninguna razón por la cual no podamos tomar esta perspectiva: primero, que para comprender cuales fueron los problemas por ejemplo, de Platón necesitamos leerlo muy cuidadosamente en el contexto de la vida griega y segundo, que cuando lo hemos leído de este modo encontraremos a menudo que estaba interesado en muchos de los 
mismos problemas que nos interesan ahora a nosotros y ofrece respuestas alternativas a ellos. ${ }^{5}$

¿Cuál es pues la comprensión histórica razonable de las filosofías del pasado? En una revisión general, el problema de las historias culturales es que no están necesariamente interesadas en las teorías filosóficas, para ellas la filosofía no es sino un síntoma más de la época en estudio. Sin embargo, como el objetivo de Passmore es hacer compatibles la perspectiva histórica y la filosófica, insiste en que no estamos obligados a acercarnos a las filosofías del pasado o bien considerando a los filósofos únicamente como "pensadores representativos del espíritu de una época" o, en el otro extremo, ignorando por completo su situación temporal. ${ }^{6}$

Como se trata de evitar estos dos extremos es muy importante caracterizar mejor la continuidad relativa de la que hemos hablado. Estrictamente, ¿qué se entiende por problemas filosóficos persistentes? ¿Acaso se trata de un conjunto de problemas invariables? Para Passmore lo que hay son tipos de problemas que los autores proponen y tratan de resolver y que tienen importancia no sólo para la cultura en la que vivieron sino para muchas otras. Esto desde luego no excluye la posibilidad del surgimiento de nuevos problemas.

Así, para hacer historia de la filosofía se debe partir de la relativa continuidad y además tener un doble enfoque, a saber: conocer la cultura de la época en la que se inscribe el pensador pára ubicarlo en ella, pero a la vez, destacar los rasgos característicos de ese autor que hacen que su obra sea interesante o importante en diversos tiempos y países. El valor de un autor no le viene únicamente por pertenecer a un determinado tiempo o cultura, sino de las aportaciones concretas que él hace a la filosofía. Así, la filosofía tiene relativa autonomía, es una investigación relativamente autónoma porque, sin perder de vista su relación con otros quehaceres culturales, tiene su propio campo. De esta manera es importante enjuiciarla como parte de una civilización pero también hacia dentro de sí misma, con respecto a sus propios logros.

En suma, ni completamente aislada de la cultura, ni completamente inmersa en su caudal, la filosofía debe ser tratada histórica y filosóficamente (crítica y culturalmente) pero sin exagerar o excluir ninguna de estas perspectivas.

En la zona intermedia que enlaza los procedimientos o métodos históricos con los filosóficos, Passmore encuentra tres alternativas a las que denomina historias elucidatorias de la filosofía. La primera es

6 Cfr. p. 15. 
la historia doxográfica que se limita a la presentación de la vida y opiniones de los autores. Tradicionalmente esta información se ordena o reordena por escuelas al margen de la crítica y de las auténticas relaciones históricas entre los autores. A pesar de sus limitaciones la historia doxográfica es punto de partida para introducirnos más a fondo en la problemática de los autores.

La segunda alternativa es la historia retrospectiva. Para ésta la historia de la filosofía no es una mera sucesión de opiniones que los historiadores deban calificar de verdaderas o falsas, sino más bien un desarrollo gradual hacia la verdad, verdad que ahora se ve como plena y final. El filósofo ya no es aquel que sostiene una opinión o adopta un punto de vista, sino un pensador que lucha por alcanzar la verdad y hace una contribución a la verdad final. Naturalmente, quedarán fuera todas aquellas tendencias del pensamiento que no puedan reabsorberse bajo la filosofía que se ha tomado como punto culminante del desarrollo.

Finalmente, la tercera alternativa que refiere Passmore es la historia problemática la cual remite a la necesidad de seguir al autor en su búsqueda de la verdad, teniendo en cuenta que el pensamiento de un autor está determinado por situaciones concretas. La historia problemática pregunta ¿qué problemas trata de resolver un filósofo? ¿cómo surge esa problemática para el filósofo? y ¿con qué nuevos métodos la maneja? Según Passmore:

...precisamente porque la historia de la filosofía puede ser concebida de esta manera, es por lo que ella misma es una tarea intelectual, una tarea además que requiere que sus expositores sean ambas cosas, historiadores y filósofos. ${ }^{7}$

Las historias problemáticas son más estudios y comentarios especializados que otra clase de historias; esto se debe a que ocupan un lugar intermedio entre la mera información doxográfica y las historias generales.

El historiador problemático no necesita ser un filósofo importante pero debe tener una comprensión simpatética de lo que es estar filosóficamente emproblemado y moverse hacia la solución del problema; una comprensión que se obtiene únicamente en la práctica filosófica. Inevitablemente (el filósofo) será desviado por su filosofía - cometerá errores que un no filósofo no cometería- pero sin ella escasamente iría más allá del nivel de la doxografia. ${ }^{8}$

\footnotetext{
7 Ibid. p. 29.

8 Ibid., p. 30.
} 
Para finalizar, me gustaría retomar el problema en mis propios términos. Mi estrategia es extender el término reconstrucción racional de manera que abarque no sólo perspectivas de la filosofía analítica sino cualquier forma de acercarse a la historia de la filosofía que implique un punto de vista filosófico, el cual puede ir desde una concepción adoptada de lo que es filosofía, que opere como guía racional mínima, hasta una teoría amplia y propia que pretenda dar cuenta, parcial o totalmente, del pasado filosófico.

Así, la reconstrucción racional puede verse desde perspectivas menos limitativas. De manera general, puede considerarse como la guía racional que forma parte del marco teórico del cual partimos y que nos sirve de hilo conductor en nuestra tarea de historiar la filosofía.

Es la historia de la filosofía la disciplina donde encarna el patrón o modelo filosófico conforme al cual se medirán las cuestiones filosóficas. La guía racional tiene así una función normativa; prescribe de entrada qué es y qué no es filosofía. Así pues, se constituye en el criterio de lo filosófico para el reconstructor de la filosofía (histórico o racional).

Además, no únicamente sirve de fundamento teórico sino que permea toda la reconstrucción en vista de que:

1) Permite la selección de autores, tópicos, escuelas, etc.

2) Nos dice si hay o no un contenido filosófico; si hay una o varias filosofías, cómo establecer sus vínculos, etc.

Bien entendido el problema, aproximar la reconstrucción racional a la histórica es darse cuenta de que por su peculiar ubicación crítica los contenidos filosóficos requieren además del tratamiento historiográfico, de un enfoque filosófico que nos permita ordenarlos, analizar sus contenidos, apreciar su desarrollo, en una palabra, darle sentido al inmenso caudal filosófico. Desde esta perspectiva, la reconstrucción racional es inseparable de la reconstrucción histórica.

El carácter creativo, crítico, etc., en la historia de la filosofía dependerá de las pautas de la reconstrucción racional, las cuales derivan directamente de una perspectiva filosófica adoptada.

\section{Metodología reconstructiva e historia de la filosofia}

Si para cualquier autor, período o escuela a historiar es fundamental contar con un concepto de filosofía que nos permita seleccionar problemas y ubicar temáticas, esto es, ordenar y valorar el caudal filosófico, el cual ha sido ya varias veces revisado y jerarquizado por los diversos historiadores de la filosofía, con mucha más razón se requiere proponer una guía racional en campos menos estudiados y más específicos como los de las filosofías regionales. 
Hay que entender que para las filosofías regionales la selección y evaluación de temas, autores, etc., es tarea fundamental ya que sabemos que no contamos con un censo de autores o un elenco de temas completo y confiable. En buena medida, nuestra tarea es justamente determinar quiénes y con qué aportaciones, han contribuido a enriquecer el caudal filosófico.

En esta tarea no se puede soslayar nuestra personal guía racional, ni la posición filosófica de la cual deriva. Sin embargo, eso no significa contaminar de subjetividad en un sentido negativo nuestra labor, sino darle unidad y sentido en función de nuestros intereses, problemas y necesidades actuales, lo cual no sólo es legítimo sino deseable desde un punto de vista creativo y critico. La guía racional no implica necesariamente deformar a los autores si se hace explícita y se muestran con claridad sus limitaciones y ventajas; en todo caso manifiesta simplemente la insoslayable ubicación concreta de cada uno de nosotros de la cual no podemos prescindir.

El marco teórico de quien hace historia de la filosofía no se puede reducir únicamente a la guía racional, debe contar además con una perspectiva historiográfica, la cual en mi concepto puede entenderse como las distintas formas de aproximación al objeto de estudio previamente constituido gracias a las pautas de la guía racional.

El objeto histórico-filosófico no es algo dado por completo, sino que se constituye mediante las pautas que nuestro enfoque filosófico nos proporciona. Este mismo enfoque condiciona la selección de estrategias metodológicas de carácter estrictamente historiográfico. Ambos aspectos constituyen el marco teórico que nos brinda un punto de vista, una forma específica de dar sentido y unidad a lo disperso, además de modos particulares de aproximación a nuestro objeto de estudio.

\section{Estrategias historiográficas de aproximación al objeto de estudio}

Quisiera iniciar esta sección con la siguiente pregunta. Si su objeto de estudio fuera el pensamiento filosófico de Don Carlos de Sigüenza y Góngora, cómo le gustaría aproximarse a él: ¿en coche de caballos, con un microscopio electrónico o desde un telescopio lunar?

Naturalmente el modo de aproximación dependerá de su preferencia filosófica, pero permítase por un momento un vacío teórico $y$ trate de visualizar ventajas y dificultades que ofrece cada una de estas formas de aproximación.

1. Si usted eligió ir en coche de caballos con Sigüenza, es indudable que conocerá el México del siglo XVII. Recorrerá con él la calle de Jesús María; le escuchará sostener barrocas y eruditas conversa- 
ciones con Sor Juana y posiblemente logre oír a esta última dedicar a nuestro amigo aquel famoso poema que comienza: "Dulce, canoro, cisne mexicano..." Acudirá en primera fila al motín del 8 de junio de 1692 y le ayudará a rescatar los archivos del Palacio Virreinal. Sabrá usted de sus deudas y desventuras. Se enterará de las multas que la Real y Pontificia Universidad tuvo que aplicarle por no acudir a impartir su lección. Compartirá su esperanza de que los reyes de España no echen en saco roto su advertencia de ocupar la Bahía de Panzacola, en vista de su ubicación estratégica. Sabrá de sus altercados con el P. Kino. Se regocijará al verle leer a Descartes y escribir con moderno aliento la Libra astronómica y filosófica. En una palabra, reconstruirá el contexto cultural. Pero vaya aún más lejos. Trate de hablar como él lo hacía y de pensar como él pensaba; lea la producción científica y filosófica que como revisor tuvo oportunidad de leer. Sin duda estará muy cerca del "espíritu filosófico" de esa época; no obstante, ¿logrará oír y hacer oír a los demás lo que verdaderamente quiso decir Sigüenza? Y por otra parte, ¿bastará la reconstrucción contextual para apreciar y comprender el pensamiento filosófico de este autor, si acaso lo hay? ¿Cómo valorar sin pautas, medidas o hipótesis derivadas de una guía racional? ¿Cómo olvidar el tiempo, el espacio, los problemas e intereses desde los cuales demandamos explicaciones? Estas y otras preguntas semejantes nos llevan a concluir que no se puede dar la reconstrucción histórica al margen de la racional.

2. Supóngase ahora que usted opta por poner a Sigüenza bajo el microscopio. Está usted en el terreno de una reconstrucción racional como la practican algunos filósofos analíticos. Someta al examen cuidadoso tesis y argumentos y decida al margen del contexto el valor científico y filosófico de la Libra. Aparte de las contradicciones, círculos viciosos y paradojas que seguramente se encuentran allí, medido con una suerte de parámetro absoluto, es posible que usted encuentre en este autor escasa aportación y nula originalidad y hasta decida que ni siquiera se trata de un filósofo. Sin buscar soslayar los errores argumentativos y las falacias ¿hasta dónde puede admitirse que para muestra basta un botón? ¿Cómo concluir sobre un todo si sólo se analizan algunas partes? El análisis, ni duda cabe, es un instrumento poderoso que permite establecer muy finas distinciones conceptuales; pero no tomar en cuenta la génesis y desarrollo de las tesis, desvincularlas del complejo de un sistema, ignorar el contexto general histórico, etc., puede llevar muy fácilmente a distorsiones y evaluaciones que se antojan impropias. Con todo, la mirada microscópica revela interesantes matices de los contenidos filosóficos que no son dables desde ninguna otra perspectiva, 
por lo cual constituye otra valiosa forma de aproximación al objeto histórico-filosófico.

3. Finalmente piense que decide ver a Sigüenza desde un telescopio ubicado en la luna. Esta amplia perspectiva que le aleja en tiempo y espacio no le deja ver las particularidades del individuo pero le permite contemplar amplios panoramas. En este caso, probablemente, las luchas colonialistas entre potencias, las manifestaciones claras de una conciencia nacional, la problemática criolla, etc., de manera dinámica. Comprenderá el sentido y ritmo propio de estos grandes movimientos, pero mientras no baje de su telescopio la especificidad de este criollo nacionalista que fue Sigüenza se le escapará.

En suma, me parece que las perspectivas aludidas ilustran algunos aspectos del problema de la relación objetivismo-subjetivismo y simplemente creo que no es posible separar perspectivas que son, en buena medida, complementarias. La historia ha menester de diversas estrategias metodológicas para una mejor comprensión y un enjuiciamiento más certero de las diversas expresiones filosóficas. Creo que por el camino por el que voy no puedo sino terminar reclamando el pluralismo metodológico para la historia de la filosofía.

\section{HISTORIA DE LA FILOSOFIA: ¿PARA QUÉ?}

Alejandro TOMASINI

INSTITUTO DE INVESTIGACIONES FILOSOFICAS, UNAM

Puede sostenerse con firmeza que, con la posible excepción de Tales de Mileto, no hay prácticamente ningún filósofo que, de uno u otro modo, no se haya apoyado en lo afirmado por sus antecesores para desarrollar sus propias ideas. Independientemente del rumbo que pueda tomar su meditación, podemos, creo, afirmar que no hay pensador que, por mínimamente que sea, no se haya refrescado el intelecto hundiéndose en el mar de pensamientos que han ido acumulándose paulatinamente y que son el material de estudio de los historiadores de la filosofía. Esto no impide que sea perfectamente concebible que la actitud de un filósofo respecto de sus antecesores sea más bien crítica o negativa. Aristóteles, por ejemplo, trata con cierta indulgencia cargada de desdén a los filósofos presocráticos, y no ve en quienes practicaron filosofía antes que él (su maestro Platón incluido) más que a los modestos preparadores de un escenario cuyo actor principal era él. Esta actitud, empero, no impide ni nulifica el hecho de que su pensamiento haya tenido que nutrirse del de ellos. 